\title{
Spectral modification of type IA fibre Bragg gratings by high power near infra-red lasers
}

Kyriacos Kalli, A. George Simpson, Kaimin Zhou, Lin Zhang, David Birkin, et al.

Kyriacos Kalli, A. George Simpson, Kaimin Zhou, Lin Zhang, David Birkin, Tim Ellingham, lan Bennion, "Spectral modification of type IA fibre Bragg gratings by high power near infra-red lasers," Proc. SPIE 6193, Reliability of Optical Fiber Components, Devices, Systems, and Networks III, 61930Y (24 May 2006); doi: 10.1117/12.662315 


\title{
Spectral modification of type IA fibre Bragg gratings by high power near infra-red lasers
}

\author{
Kyriacos Kalli ${ }^{1 *}$, A George Simpson ${ }^{2}$, Kaimin Zhou ${ }^{2}$, Lin Zhang ${ }^{2}$, David Birkin ${ }^{2}$, Tim Ellingham ${ }^{2}$ \\ and Ian Bennion ${ }^{2}$ \\ ${ }^{1}$ Higher Technical Institute, C. Kavafi Street, Nicosia, 2152 Cyprus \\ 2 Photonics Research Group, Aston University, Birmingham B4 7ET United Kingdom
}

\begin{abstract}
We report the first experimental measurements on the spectral modification of Type IA fibre Bragg gratings, incorporated in an optical network, which result from the use of high-power, near infrared lasers. The fibre grating properties are modified in a controlled manner by exploiting the characteristics of the inherent $1400 \mathrm{~nm}$ absorption band of the optical fibre, which grows in strength during the Type IA grating inscription. If the fibre network is illuminated with a high power laser, having an emission wavelength coincident with the absorption band, the Type IA centre wavelength and chirp can be modified. Furthermore, partial grating erasure is demonstrated. This has serious implications when using Type IA gratings in an optical network, as their spectrum can be modified using purely optical methods (no external heating source acts on the fibre), and to their long-term stability as the grating is shown to decay. Conversely, suitably stabilised gratings can be spectrally tailored, for tuning fibre lasers or edge filter modification in sensing applications, by purely optical means.
\end{abstract}

Keywords: Optical fibre sensors, fibre Bragg gratings, type IA grating, photosensitivity, wavelength tuning

\section{INTRODUCTION}

Type IA fibre Bragg gratings may be considered a subtype of Type I gratings and are typically formed after the prolonged UV exposure of a standard grating in hydrogenated germanosilicate fibre $\left.{ }^{1,2}\right]$, although recent improvements in their inscription have shown that they can be readily inscribed in a suitably prepared optical fibre ${ }^{3}$. The spectral characteristics of Type IA gratings are unique; they are distinct from other grating types as they exhibit a large increase in the mean core index that is identifiable as a large red shift seen in the Bragg wavelength $\left(\lambda_{B}\right)$ of the grating during inscription, figure 1 . We observe that the mean wavelength change is characterised by three distinct regimes, with the Type 1 grating growth being superseded by a quasi-linear region followed by saturation. This saturated red shift is dependent on fibre type and hydrogenation conditions, but for a highly doped fibre (either high Ge dopant or B/Ge codoped fibre) is typically in the order of $15-20 \mathrm{~nm}$, and for SMF-28 is $5-8 \mathrm{~nm}$. The maximum wavelength shift translates to an increase in the mean index of up to $2 \times 10^{-2}$. More importantly, IA gratings have been shown to exhibit the lowest temperature coefficient of all grating types reported to date, which makes them ideal for use in a temperature compensating, dual grating sensor, as has recently been demonstrated ${ }^{4}$.

Figure 2 shows the spectrum of a 4-mm regenerated IA grating and a 1-mm standard Type I grating. These gratings have been written in the same fibre with the same phase mask, yet their central reflecting peaks are $14.5 \mathrm{~nm}$ apart after annealing. The large increase in mean refractive index increases the fibre NA to such an extent that it becomes fewmoded; the NA of the virgin fibre is 0.161 , compared to 0.247 for the modified fibre, accounting for the transmission spectrum ghost mode. Type IA gratings are inscribed using the standard scanning-phase mask technique, and the fibre is illuminated with a cw UV laser source operating at $244 \mathrm{~nm}$ (Coherent SabreFreD). A mirror is mounted onto an air bearing translation stage to enable the laser beam to be scanned along the fibre length at $0.25 \mathrm{~mm} / \mathrm{s}$ and with $200 \mathrm{~mW}$ of optical power delivered to the fibre; a 1-mm aperture ensures an accurate top-hat exposure profile along the fibre length. The grating manufacture has a two-step process; first the phase mask is removed and the UV beam scanned along the fibre length to give a blank beam exposure. The mask is then reintroduced and a grating written into the UV pre-exposed section of fibre. This has proven the most successful way of inscribing Type IA gratings with a clean wavelength spectrum, as confirmed in figure 2. The fibre undergoes typical hydrogenation conditions of $80^{\circ} \mathrm{C}$ at $200 \mathrm{Bar}$ for more

\footnotetext{
Reliability of Optical Fiber Components, Devices, Systems, and Networks III, edited by Hans G. Limberger, M. John Matthewson,

Proc. of SPIE Vol. 6193, 61930Y, (2006) - 0277-786X/06/\$15 - doi: 10.1117/12.662315
}

Proc. of SPIE Vol. $619361930 Y-1$ 
than 90 hours, thereby exceeding the equilibrium time for hydrogen saturation in the fibre. The concentration of hydrogen molecules in the optical fibre core at saturation (the equilibrium solubility) exceeds $11500 \mathrm{ppm}$.

There have been earlier studies of UV pre-exposure in hydrogenated, highly doped optical fibres; see, for example, Kawano et $a l^{5}$, Chen et $a l^{6}$, Lancry et $a l^{7}$, Canning et $a l^{8}$, Mizunami et al ${ }^{9}$, MacPherson et al ${ }^{10}$, and Simpson et al ${ }^{11}$. However, none of these papers detail the effects of high optical powers on UV pre-exposed and hydrogenated optical fibres. In this work we have focussed on such effects. We report the spectral modification of Type IA fibre Bragg gratings, incorporated in an optical network, which result from the use of high-power, near infrared lasers. The fibre grating properties are modified in a controlled manner by exploiting the characteristics of the inherent $1400 \mathrm{~nm}$ absorption band of the optical fibre. If the fibre network is illuminated with a high power laser, having an emission wavelength coincident with the absorption band, the Type IA centre wavelength and chirp can be modified. Moreover, partial grating erasure is demonstrated. The highly doped fibre used in this study was Fibercore PS1250/1500 B/Ge (codoped) with NA of 0.13 . The actual dopant levels remain undisclosed.

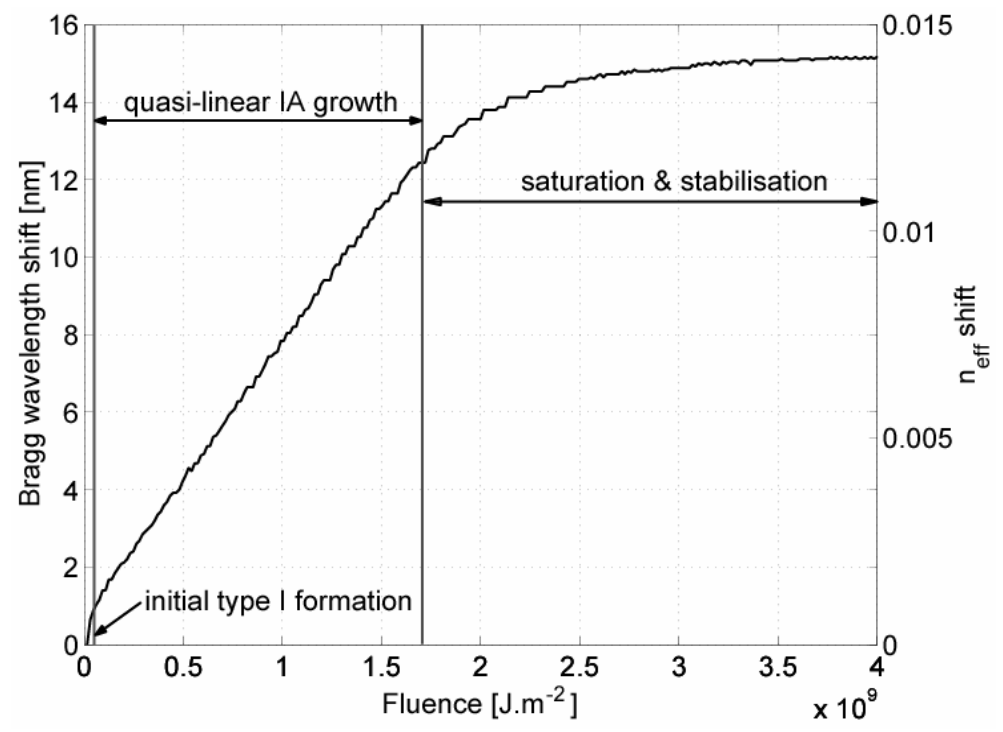

Figure 1. The characteristic red shift displayed during prolonged UV exposure of hydrogenated germanosilicate fibre, shown in terms of the spectral red shift and associated refractive index change. The three distinct regions of IA growth are shown. 


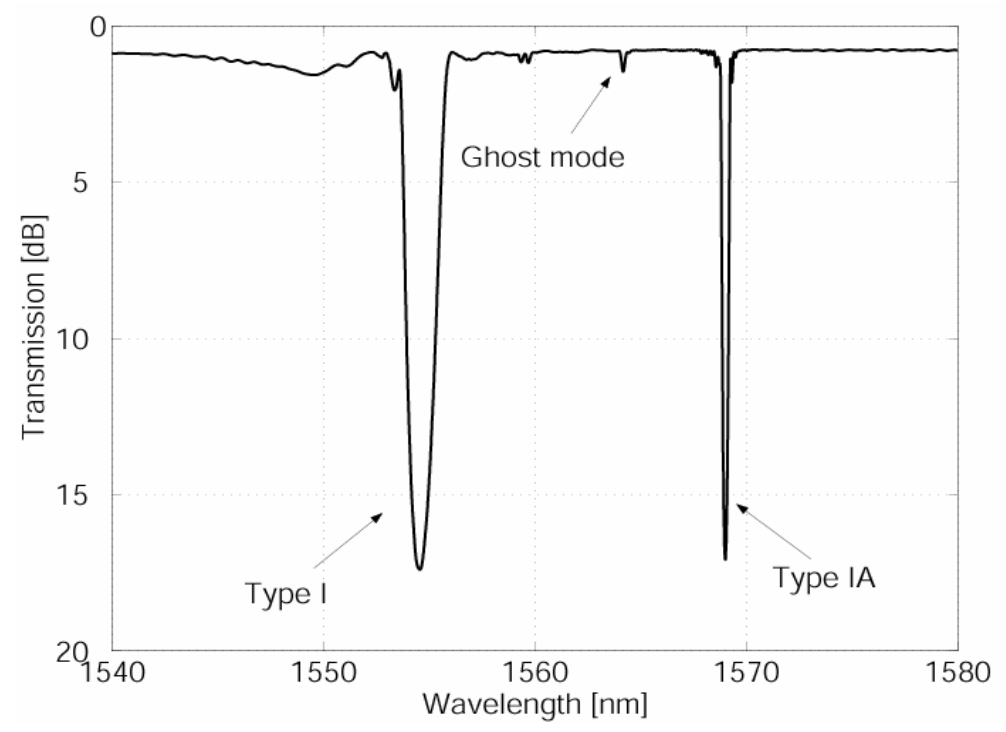

Figure 2. The spectrum of a standard grating (left) and a physically longer Type IA grating (right), written in the same fibre with the same phase mask. The 14.5-nm difference in wavelength is caused by the large increase in mean refractive that accompanies IA grating growth. This also accounts for the ghost mode, as the mean index change is sufficient to cause the fibre to become few-moded.

We have previously shown that there is a strong correlation between the growth of the $\mathrm{OH}$ absorption band formation in the optical fibre during prolonged UV exposure and the increase in the mean index change of the fibre grating ${ }^{3}$. The data in figure 3 shows the growth of a typical regenerated Type IA grating; in this case the phase mask is not removed during the manufacturing process so that the grating peak can be monitored. However, this does not produce a clean grating as the growth follows a complex process of inscription and erasure as the Bragg condition is alternately matched and mismatched throughout the UV exposure. This results from a non-uniform dc index of refraction change across the grating length.

Figure 3 confirms the parallel mean index evolution, akin to the maturity level of a Type IA grating, and the associated increase in the absorption band at $1400 \mathrm{~nm}$. Both curves follow the three trends of figure 1 . This absorption band is a consequence of the formation of $\mathrm{OH}$ ions within the fibre and has no dependence on the modulated index change required for a Bragg grating to be formed in the core ${ }^{12}$. It is therefore possible to monitor the transmission loss at $1400 \mathrm{~nm}$ and glean accurate feedback as to the maturity of the fibre. When the absorption loss saturates or the rate of change has substantially decreased, the fibre is fully mature and ready for inscription of any form of IA grating, be it slanted, long period or standard. Although not completely saturated, we have found that there is little or no effect on the actual Type IA grating once this initial fast rate of change has been exceeded - beyond 1200 seconds. We note that the peak absorption band loss at $\sim 1400 \mathrm{~nm}$ is typically $\sim 1.2 \mathrm{dBmm}^{-1}$ for a $\mathrm{B} / \mathrm{Ge}$ co-doped fibre. It is this increase in local absorption, coincident with the position of the Type IA grating, which is exploited to heat the fibre. Therefore, if the fibre is pumped with a laser source that coincides with the $1400 \mathrm{~nm}$ absorption band the fibre will be heated in the region where there is light absorption, e.g. the location of the Type IA grating. 

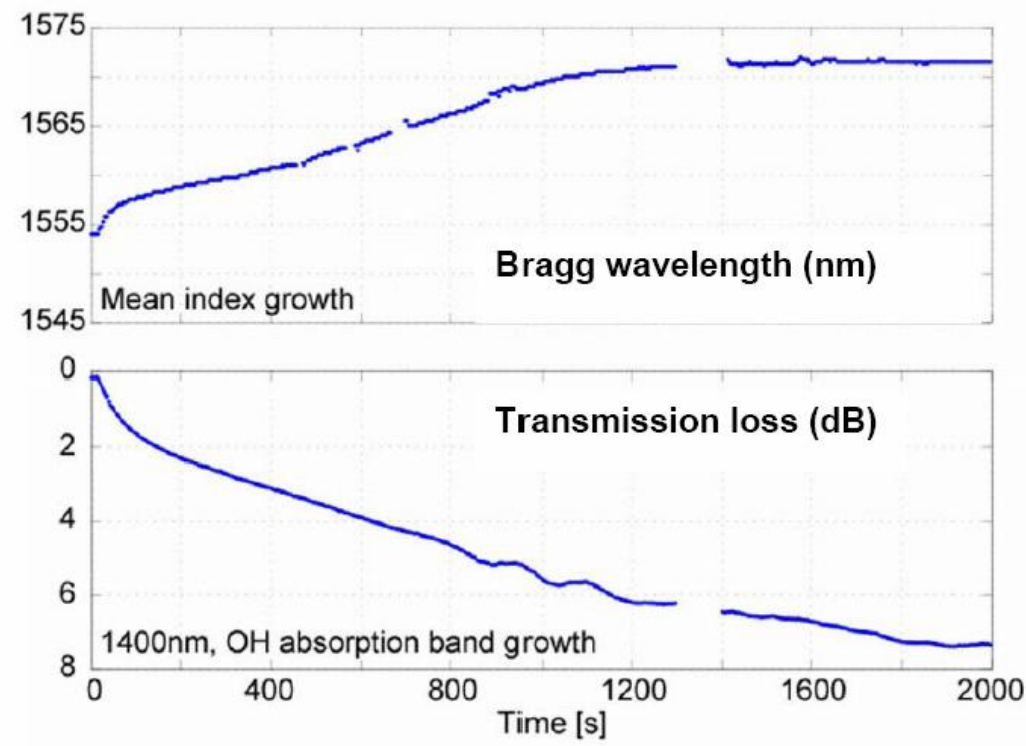

Figure 3. The relationship between the mean index change in nm (or IA maturity) as a function of time and the growth of the peak of the $1400 \mathrm{~nm}$ absorption band under UV exposure. It is this relationship which enables IA index changes to be inscribed without writing a grating since the index change may be monitored by observing the absorption band at $1400 \mathrm{~nm}$.
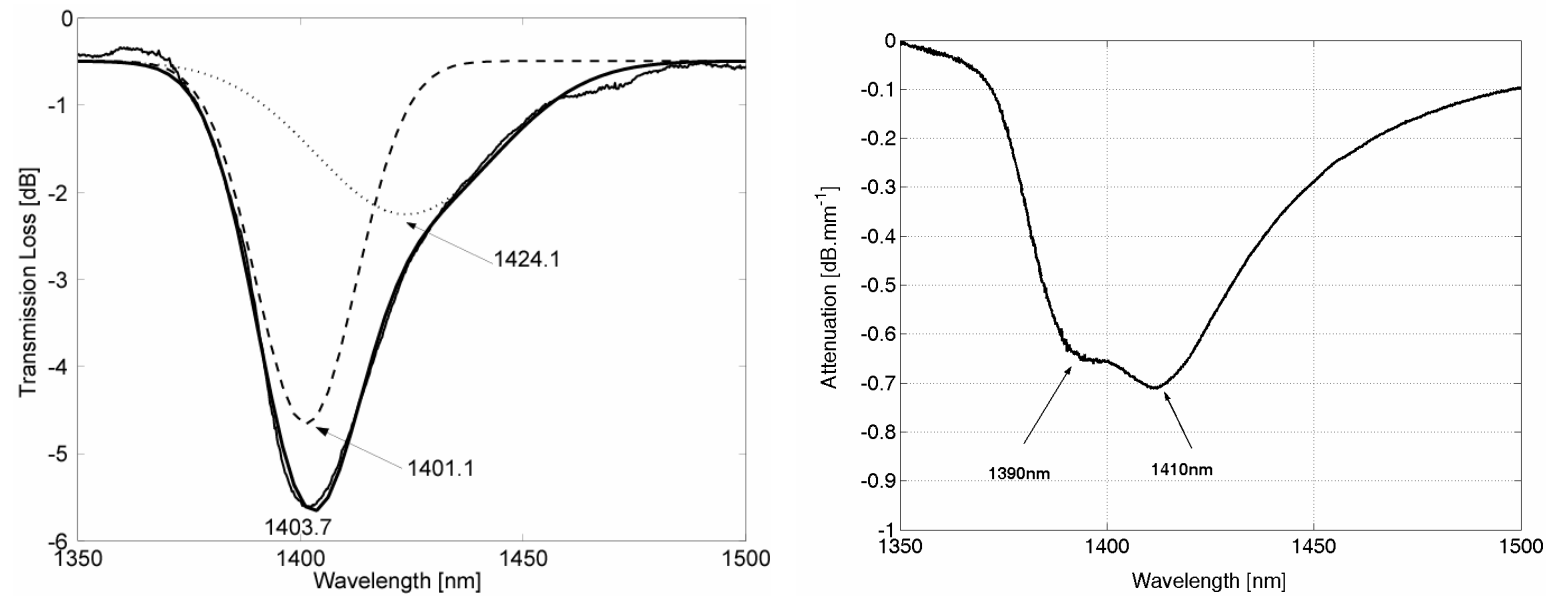

Figure 4. (a) (left) The absorption band at $1400 \mathrm{~nm}$ for the Fibercore B/Ge fibre deconvolved to show its constituent peaks - a mixture of bands - centred at $1401 \mathrm{~nm}$ and $1424 \mathrm{~nm}$. (b) (right) A typical spectrum for highly Ge-doped optical fibre (without boron) clearly showing the conventional absorption features of $\mathrm{SiOH}(1390 \mathrm{~nm}) \& \mathrm{GeOH}(1410 \mathrm{~nm})$ under similar UV exposure conditions.

In figure 4 we show the absorption features at $1400 \mathrm{~nm}$ for the Fibercore $\mathrm{B} / \mathrm{Ge}$ fibre, these are deconvolved to show its constituent peaks - a mixture of bands - centred at $1401 \mathrm{~nm}$ and $1424 \mathrm{~nm}$. We note that there is a strong suppression of the $\mathrm{SiOH}$ band at $1390 \mathrm{~nm}$. This should be compared with a typical spectrum for highly Ge-doped optical fibre (without boron) clearly showing the conventional absorption features of $\mathrm{SiOH}(1390 \mathrm{~nm})$ \& GeOH $(1410 \mathrm{~nm})$ under similar UV exposure conditions. As the Fibercore fibre is subject to confidentiality one cannot elucidate the exact dopant contributions to this absorption feature, although from a practical point of view during grating inscription one monitors the peak and stops the blank UV exposure once the growth has rolled off. The loss generated by the UV exposure is broadband hence it appears that the baseline is not coincident with zero dB. 


\section{REVERSIBLE WAVELENGTH TUNING}

To test our hypothesis of selective grating spectral modification, we utilised the network as highlighted in figure 5. A Type IA - Type I dual grating sensor was manufactured, as outlined in reference 4 and in the introduction, with the Type I grating used as a reference to subsequent measurements. The IA-I grating consisted of a 2-mm IA grating, manufactured by the blank beam UV pre-exposure method directly adjacent to a 2-mm Type I grating. The network was illuminated with an amplified spontaneous emission broadband source (ASE-BBS) in the spectral region of the gratings and monitored in reflection by the optical spectrum analyser (OSA). The data was recovered from the OSA with a computer, and subsequent data was analysed with a centroid-fitting algorithm designed to accurately track the wavelength changes. Powerful tuneable lasers were used to coincide with the $1400 \mathrm{~nm}$ absorption band. Two lasers were chosen for Type IA modification; the first was a $10 \mathrm{~mW}$ Santec laser with a range of $1410 \mathrm{~nm}$ to $1490 \mathrm{~nm}$, set to coincide with the peak of the absorption band. The second laser was a $5 \mathrm{~W}$ Raman pump operating at $1425 \mathrm{~nm}$, sitting at the edge of the band and coincident with the feature at $1424 \mathrm{~nm}$, figure 4(a).

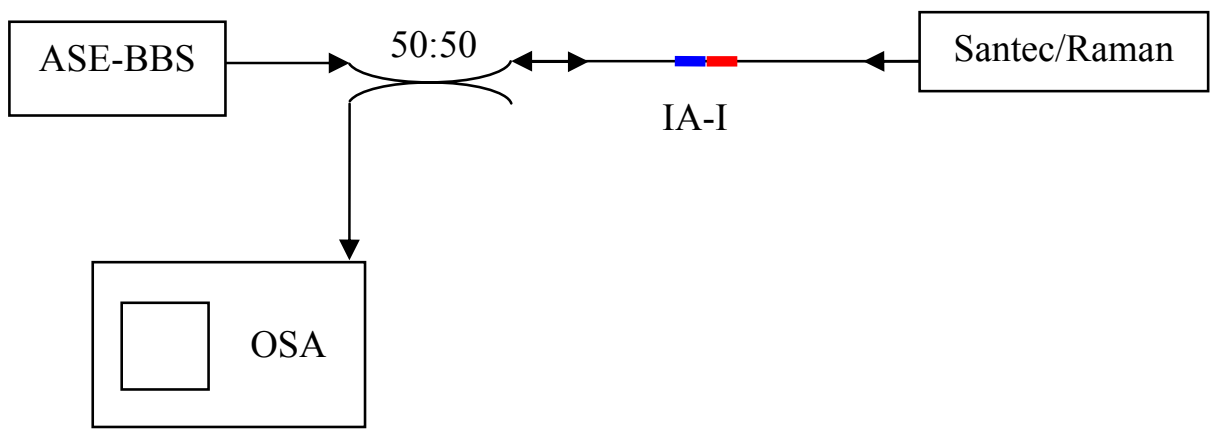

Figure 5. Experimental set-up used for Type IA laser-based modification. ASE-BBS - amplified spontaneous emission broadband source and OSA - optical spectrum analyser. Santec/Raman refers to the near infrared lasers used to illuminate the network.

\subsection{Type IA wavelength tuning - low power, near absorption peak}

Figure 6 shows the shift in $\lambda_{B}$ of the Type IA grating with increasing laser power at $1410 \mathrm{~nm}$, a wavelength that coincides with the peak of the absorption band, and demonstrates that even for modest optical powers significant wavelength offset can be induced. This could prove to be a deleterious for gratings used as narrow band optical filters in multi-wavelength systems. The demonstrated $100 \mathrm{pm}$ wavelength shift would change the grating's spectral response adversely affecting the filter performance, and reducing the isolation between wavelength channels, transmission properties and effective bandwidth. There was no measurable change in the centre wavelength of the adjacent Type I grating, which is expected as the absorption band is located at the position of the Type IA grating alone.

Unfortunately a limiting factor in this data set is the minimum resolvable wavelength change of the OSA, as shown by the pixelated data. In figure 7 the wavelength of the tuneable laser is shifted (1410nm to 1490nm) whilst maintaining a constant output power $(10 \mathrm{~mW})$ and the shape of the $\mathrm{OH}$ band is used as an edge filter, varying the amount of energy absorbed and hence the tuning of $\lambda_{\mathrm{B}}$. The pixelated data again results from the limited OSA resolution. 


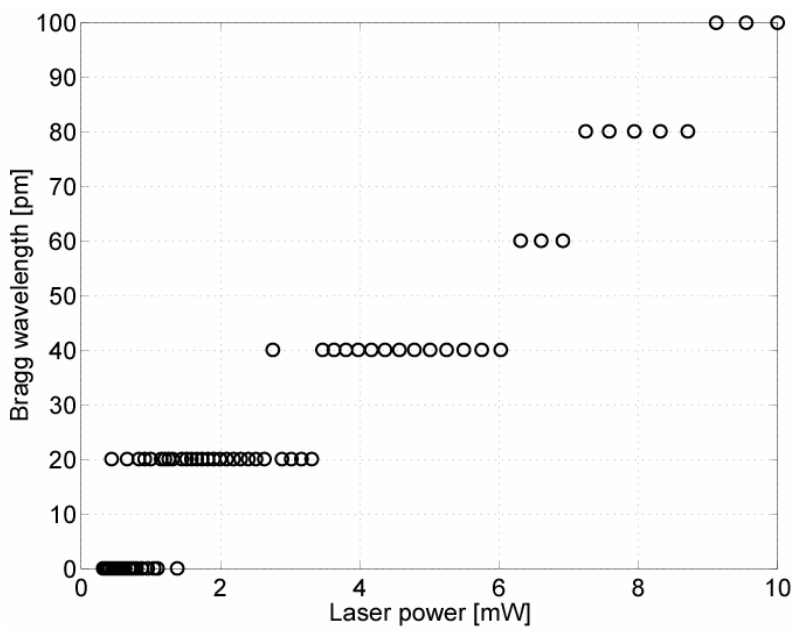

Figure 6. The shift in the Bragg wavelength of a IA grating heated with a 1410nm tuneable laser of varying power levels. This shows that the Bragg wavelength of a Type IA grating may be controlled remotely with strictly optical means. The pixelated data results from the OSA resolution limitation.

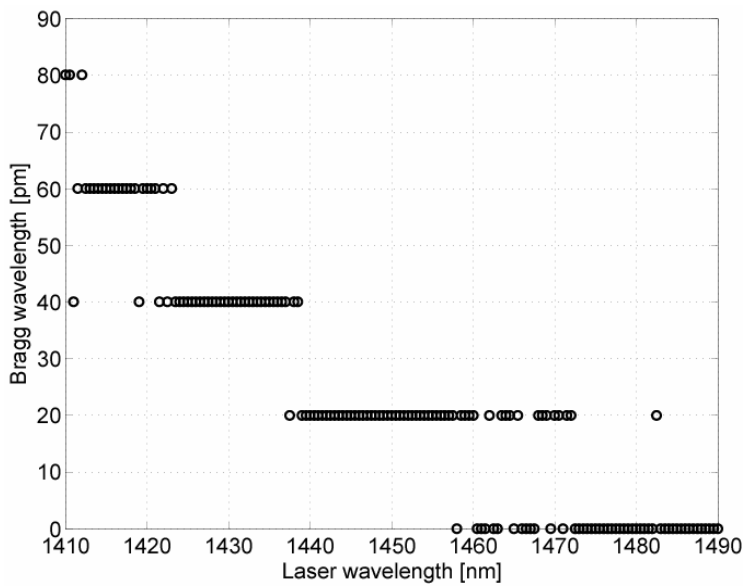

Figure 7. The shift in the Bragg wavelength of the Type IA grating when the tuneable laser was held at a constant power (10mW) and the wavelength was shifted up the edge of the $\mathrm{OH}$ band. This further shows that the Bragg wavelength of a Type IA grating may be controlled remotely with strictly optical means. The pixelated data results from the OSA resolution limitation.

\subsection{Type IA wavelength tuning - high power, far from absorption peak}

The experiment was reconfigured so that the high power Raman source, operating at $1425 \mathrm{~nm}$ and far from the main absorption peak, illuminated the Type IA-I gratings. There was no measurable change in the centre wavelength of the adjacent Type I grating that could be attributed to the pumping with the Raman source, and small shifts were attributed to fluctuations in the ambient temperature over the duration of the experiment. Although the gratings were directly adjacent to one another, the fibre portion that was pre-exposed to UV radiation and contains the higher wavelength IA grating only absorbed the $1425 \mathrm{~nm}$ radiation; the Type I grating written in virgin fibre with no strong $1400 \mathrm{~nm}$ absorption band was not heated. The negligible thermal mass of the fibre prevented any significant levels of thermal conduction between the two regions. Thus the application of the Raman laser caused the Type IA grating to spectrally shift whilst the Type I grating maintained a fixed wavelength, again making an optically tuneable grating pair. Table 1 summarises the relative shift of gratings. Figure 8 indicates a linear relationship between Raman power and wavelength shift. 
Table 1. Wavelength shifts of the Type I and IA gratings when heated with a Raman source at $1425 \mathrm{~nm}$.

\begin{tabular}{ccc}
\hline Raman Power & $\Delta \lambda_{B R}^{I A}$ & $\Delta \lambda_{B R}^{I}$ \\
$\mathrm{~mW}$ & $\mathrm{pm}$ & $\mathrm{pm}$ \\
0 & 0 & -3 \\
25.1 & 50 & 0 \\
52.9 & 100 & 11 \\
79.22 & 160 & 11 \\
103.45 & 210 & 5 \\
124.8 & 260 & 11 \\
\hline
\end{tabular}

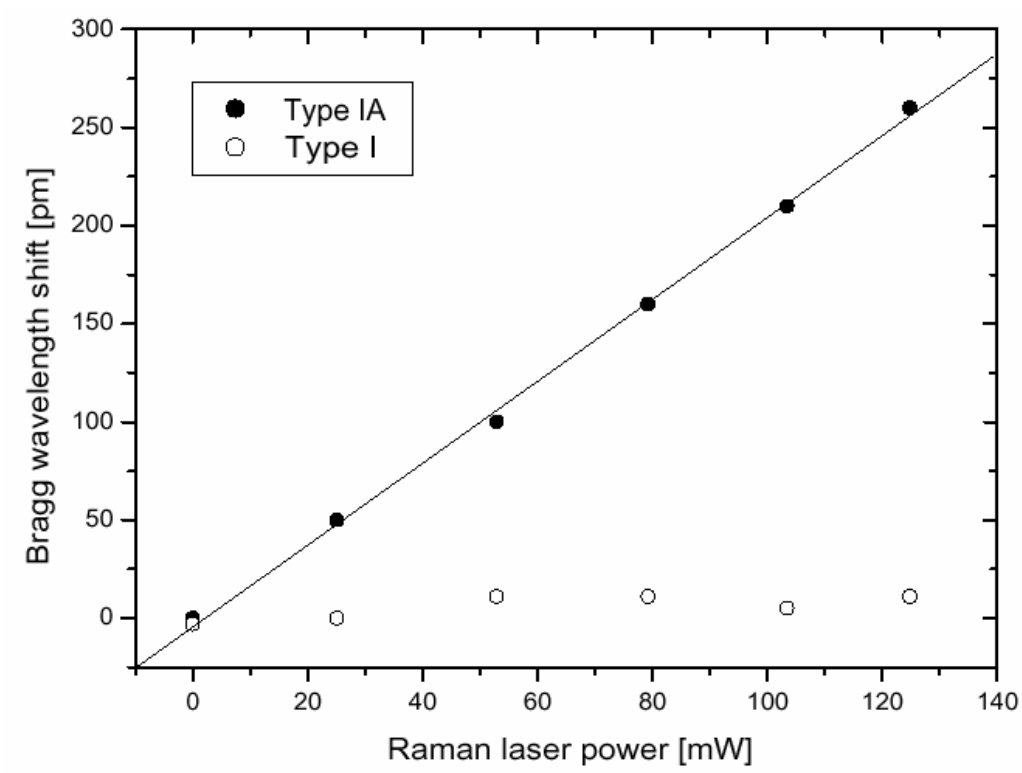

Figure 8. A plot of the wavelength shifts of the Type I and IA gratings when heated with a Raman source at $1425 \mathrm{~nm}$. This figure clearly shows a controllable change in the Bragg wavelength of the Type IA grating and a constant Bragg wavelength of the Type I grating.

\section{INDUCING CHIRP AND PERMANENT, PARTIAL GRATING DECAY}

If we next consider the effect of the Raman source being absorbed along the length of the Type IA grating we note that the heating effect will be non-uniform along the length of the absorbing section of fibre and that the result will be an induced chirp along the grating. Clearly higher Raman powers will induce a larger chirp and we should see an increase in the full width at half maximum (FWHM) reflectance bandwidth. None was noted with the lower power source. The Raman source intensity decay along the Type IA grating length is given by

$$
I(p)=I(p-1) \cdot\left(1-10^{\frac{A}{10}}\right) \propto \Delta T
$$


where $I(p)$ is the Raman intensity at a point $p, A$ is the absorption per unit length of the Raman radiation measured in $\mathrm{dB}$ and $\Delta T$ is the induced temperature increase. Figure 9 (a) shows this relation for the power levels used to create figure 9 (b) and an estimation of the absorption coefficient of $1.2 \mathrm{dBmm}^{-1}$. Table 2 summarises this data and details both a clear increase in the Bragg wavelength and the FWHM bandwidth of the reflectance peak.
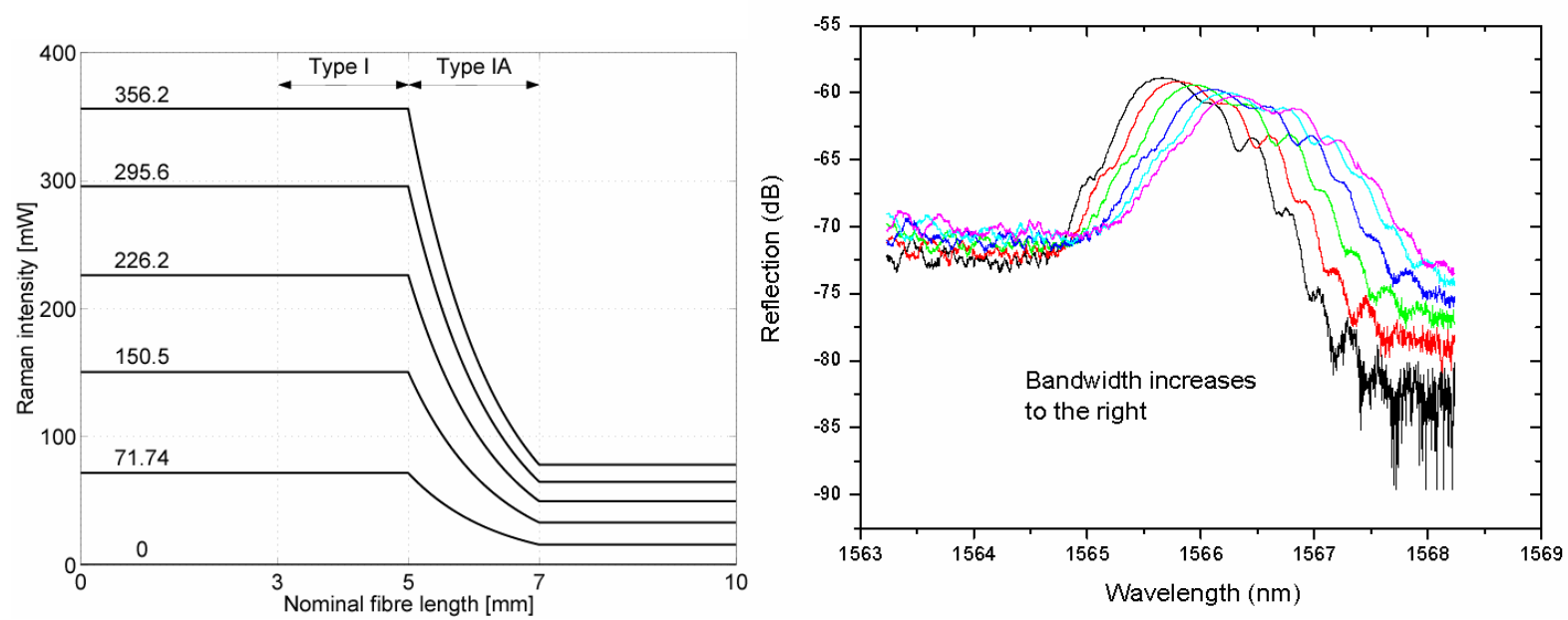

Figure 9. (a) (left) A simulation showing the decay in the intensity of the Raman laser along the length of a 2-mm IA grating. (b) (right) The overlaid reflection spectra of the Type IA grating under the heating effect of a Raman laser. The bandwidth increases sequentially from 1.31 to $1.73 \mathrm{~nm}$, as detailed in Table 2. This figure shows that it is also possible to tune the chirp of the Type IA grating by purely remote optical means.

Table 2. Summary of the wavelength shift and FWHM expansion of the Type IA grating when heated with a Raman source at $1425 \mathrm{~nm}$.

\begin{tabular}{c|cc}
\hline Raman Power & $\Delta \lambda_{B R}^{I A}$ & $\mathrm{FWHM}$ \\
$\mathrm{mW}$ & $\mathrm{pm}$ & $\mathrm{nm}$ \\
0 & 0 & 1.31 \\
71.74 & 160 & 1.36 \\
150.5 & 330 & 1.45 \\
226.2 & 520 & 1.55 \\
295.6 & 660 & 1.65 \\
356.2 & 770 & 1.73 \\
\hline
\end{tabular}

The data of Table 2 is plotted in figure 10, indicating linear relationships between the FWHM bandwidth and the Raman intensity (figure 10 (a)) and also the Bragg wavelength shift and Raman intensity (figure 10 (b)). The data of figure 8 is added to show that the wavelength shift is consistent for both high and low powers, and indicates that the wavelength shifts are due to purely temperature induced changes via optical absorption of the pumping laser source. The temperature change associated with the observed wavelength shift is added as an upper axis to figure 10 (b), and is based on assuming a temperature to wavelength responsivity of approximately $8 \mathrm{pm} /{ }^{\circ} \mathrm{C}$ for a Type IA grating in this fibre type ${ }^{3}$. 

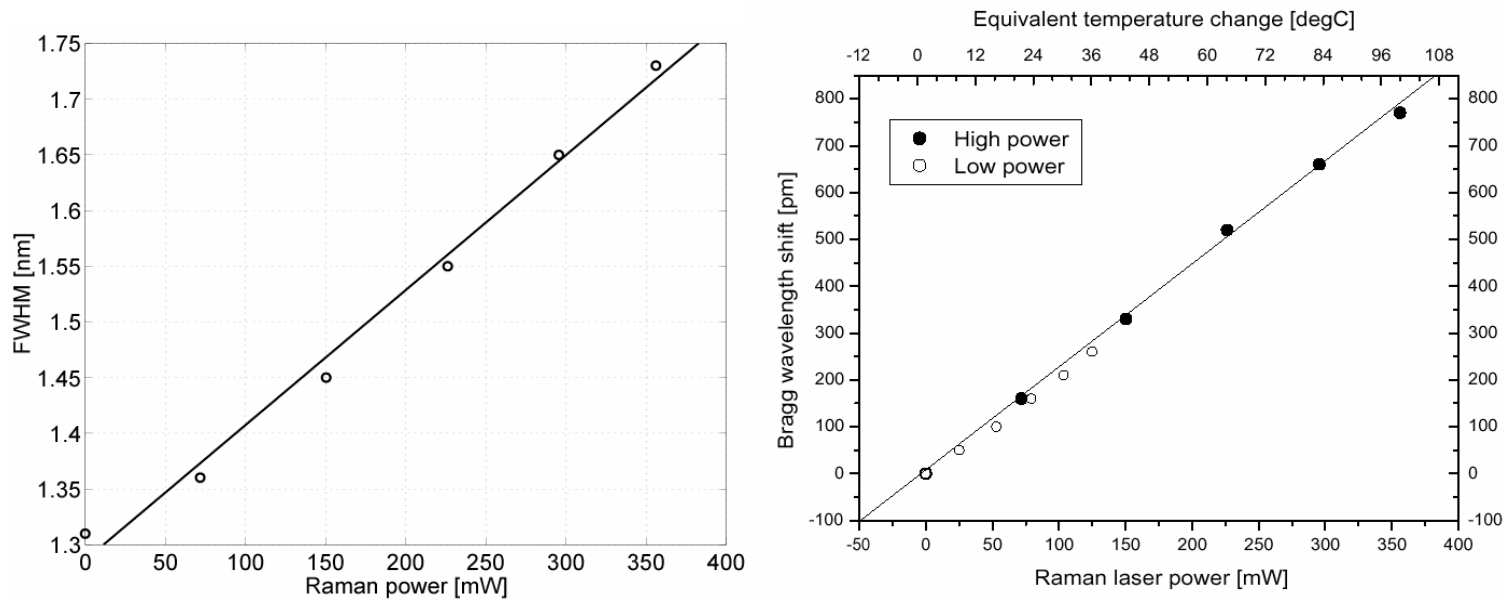

Figure 10. (a) (left) A plot showing the linear relation between the FWHM bandwidth of the Type IA grating and the intensity of the Raman laser. (b) (right) A plot showing the linear relation between the Bragg wavelength shift of the Type IA grating and the intensity of the Raman laser.

Finally, in figure 11, we observe that under continued laser pumping at $1425 \mathrm{~nm}$ and high power, one can induce partial but permanent erasure of the modulated grating component. This is again a thermally induced effect that results from local absorption of the Raman pump source, in the vicinity of the Type IA grating. Given the controlled nature and online monitoring it is important to note that this approach could be used to anneal a Type IA grating during the grating inscription process.

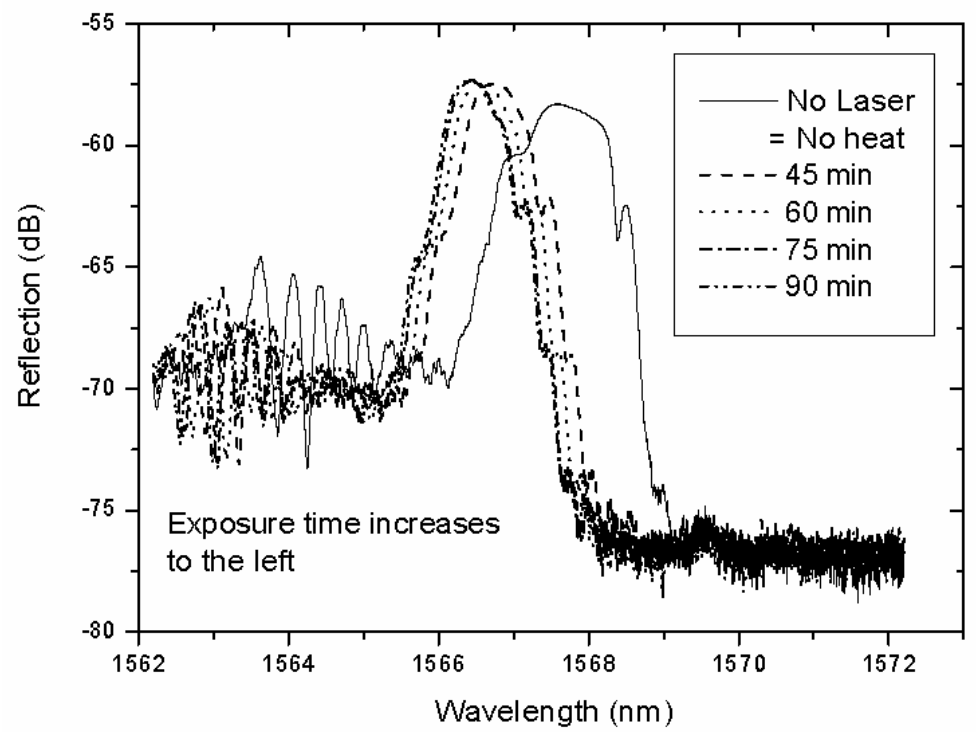

Figure 11. Data represents the permanent, and partial, laser-induced erasure of the Type IA grating with time. The grating wavelength is seen to shift to lower wavelengths (to the left) under continued exposure to pumping laser power (and hence local heating). 


\section{CONCLUSIONS}

We have shown that it is possible to selectively heat and modify the spectral characteristics of a grating within an array of other gratings by purely optical methods, in this case using two different laser sources operating in the near infra-red. The first laser source has a low power but coincides with the absorption peak of the characteristic $1400 \mathrm{~nm}$ fibre absorption band, and induces small but significant wavelength shifts of approximately $100 \mathrm{pm}$ at $10 \mathrm{~mW}$ power. The second laser operating at $1425 \mathrm{~nm}$ and far from the absorption peak induces wavelength shifts in excess of $750 \mathrm{pm}$ and a $30 \%$ increase in FWHM for a pump power of $350 \mathrm{~mW}$. We have induced reversible wavelength shifts, chirp and controlled permanent grating decay (annealing). This has serious implications when using Type IA gratings in an optical network, as their spectrum can be modified using purely optical methods (no external heating source acts on the fibre), and to their long term stability as the grating is shown to decay. We note that high power lasers are increasingly being used in optical networks and our study may have greater implications to all grating types, as laser powers and the useable wavelength spectrum increase. This may result from the presence of absorption bands in the visible and near infra-red that are produced due to the fibre being pre-conditioned, prior to grating inscription (as in this case). Absorption features are notable by their presence at shorter wavelengths for conventional Type I gratings inscribed in hydrogenated fibre. Whereas these absorption features are not typically considered to be of consequence to grating lifetime their impact has yet to be conclusively established. This is being examined as an on-going activity of the COST270 action "Reliability of Optical Components and Devices in Communications Systems and Networks" ${ }^{13}$.

Conversely, there are applications where suitably stabilized Type IA gratings can be spectrally tailored, for tuning fibre lasers or edge filter modification in sensing applications. The latter results from the non-uniform absorption of the pumping laser source at it traverses the Type IA grating, and as demonstrated in the data of figure 9 (b). The fact that this type of spectral tuning can be realized through the use of an additional laser source can be advantageous as no special coatings to the fibre are necessary, and all degrees of tuning can be set during the grating manufacturing process thereby offering great flexibility at the design stage. Since all grating types can be written in a section of pre-exposed fibre this method of optical tuning could be used for all existing Bragg grating applications making the technique invaluable to a multitude of applications. Finally we note that it is possible to tailor the absorption of the pre-exposed section to mirror the decay in intensity resulting in a uniform heating of the grating. However, this would alter the mean fibre index along the pre-exposed section, inducing a potentially large (up to $20 \mathrm{~nm}$ ) chirp across the grating.

\section{ACKNOWLEDGEMENTS}

The authors acknowledge the UK DTI-EPSRC LINK project EMPIRE. KK and AGS respectively acknowledge the Higher Technical Institute - Promotion of Research Fund and studentship of the UK EPSRC and BAE Systems.

\section{REFERENCES}

1. Y. Liu, J.A.R. Williams, L. Zhang and I. Bennion, 2002 Abnormal spectral evolution of fibre Bragg gratings in hydrogenated fibres Opt. Lett. 27 586-588.

2. A.G. Simpson, K. Kalli, K. Zhou, L. Zhang and I. Bennion, 2004 Formation of type IA fibre Bragg gratings in germanosilicate optical fibre Elec. Lett. 40 163-164.

3. A.G. Simpson, K. Kalli, L. Zhang, K. Zhou and I. Bennion, 2003 Abnormal photosensitivity effects and the formation of type IA FBGs, BGPP, Monterey, California, MD31.

4. A.G. Simpson, K. Kalli, K. Zhou, L. Zhang and I. Bennion, 2003 An idealised method for the fabrication of temperature invariant IA-I strain sensors, postdeadline session, OFS-16 Nara, Japan, PD4.

5. H. Kawano, H. Muentz, Y. Sata, J. Nishimae and A. Sugitatsu 2001 Reduction of transmission spectrum shift of long-period fiber gratings by a UV pre-exposure method J. Lightwave Technol. 190 1221-8

6. K. P. Chen, P. R. Herman and R. Tam 2002 Strong fibre Bragg grating fabrication by hybrid 157- and 248-nm laser exposure IEEE Photon. Technol. Lett. 14 170-2

7. M. Lancry, P. Niay, S. Bailleux, M. Douay, C. Depecker, P. Cordier and I. Riant 2002 Thermal stability of the 248$\mathrm{nm}$-induced presensitization process in standard H2-loaded germanosilicate fibres Appl. Opt. 41 7197-204

8. J. Canning, M. Aslund and P-F. Hu 2000 Ultraviolet-induced absorption losses in hydrogen-loaded optical fibres and in presensitized optical fibres Opt. Lett. 25 1621-3 
9. T. Mizunami, T. Fukuda and A. Hayashi 2004 Characterization of long-period-grating temperature sensors using GeB-co-doped photosensitive fibre and single-mode fibre Meas. Sci. Technol. 15 1467-1473

10. W.N. MacPherson, R.R.J. Maier, J.S. Barton, J.D.C. Jones, A. Fernandez Fernandez, B. Brichard, F. Berghmans, J.C. Knight, P.StJ. Russell and L. Farr 2004 Dispersion and refractive index measurement for Ge, B-Ge doped and photonic crystal fibre following irradiation at MGy levels Meas. Sci. Technol. 15 1659-1664

11. G. Simpson, K. Kalli, K. Zhou, L. Zhang and I. Bennion 2004 Blank beam fabrication of regenerated type IA gratings Meas. Sci. Technol. 15 1665-1669

12. P.J. Lemaire, R.M. Atkins, V. Mizrahi and W.A. Reed, 1993 High-pressure H-2 loading as a technique for achieving ultrahigh UV photosensitivity and thermal sensitivity in GeO2 doped optical fibres Elec. Lett. 29 1191-1193.

13. See www.cost270.com and contents therein.

*kkalli@cytanet.com.cy; phone 35722406537; fax 35722406545 